التعاون المائي بين الاردن و إسر ائيل ومعاهدة

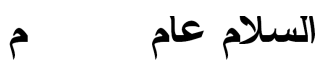

خالدة إبراهيم خليل الحبيطي*

لقد كانت مشكلة المياه ومازالت، احدى الآسباب الرئيسة لتوتر العلاقات بين

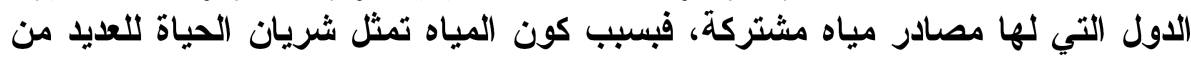

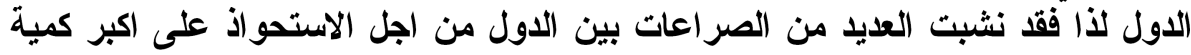
من مصادر المياه.

وقد شهلت منطقة الثرق الأوسط والوطن العربي خاصة صراع بين الدول

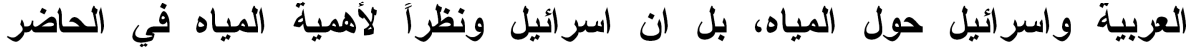

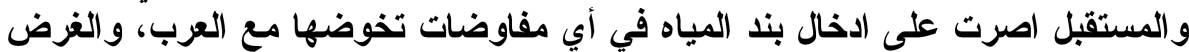

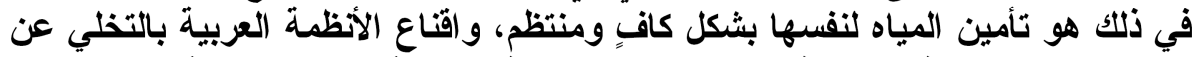

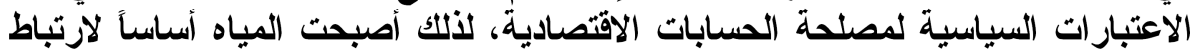
اسر ائيل عضوياً بالشرق الأوسط و الندماجها في البيئة العربية.

المقدمها

تعتبر مشكله المياه في الثرق الاوسط من المشكلات الكبرى الخطرة التي

يتوفف عليها مصير السلام والاستقرار في المنطقه. وهي إلى جاتب كونها مساله سياسيه وا(فتصاديه وهاتونيه، تمتل مشكله تتلاخل مع مشكلات اخرى نتجت عنها وتتقاطع معها، كالاحتلال العسكري وإنشاء المستعمرات. وتاتي الانقايهه الاردنيه ـ الإسرائيليه حول المياه عام 199، لتوكد مخاطر القرار العربي المنقرد في مجال الهتسام المياه، إد ان التسويات المائيه تبقى في نهايه الامر جزءا لا يتجزا من الترتيبات الإعليميه، وض كرست الأفايه الاردنيه ـ الإسرائيليه الدور المسيطر لإسرائيل من هن مصادر المياه على طول الحدود المشتركه بين البلاين نهر الاردن، ونهر اليرموك وسيول وادي عربه. 
قالمياه في الثرق الاوسط والوطن العربي اصبحت شريان المياه ورهان المستقبل، وان إسرائيل، من بين كيانات المنطقه وهي وحدها التي استطاعت استشراق المستقبل ووضع المخططات الخاصه لخدمه مصالحها وتوفير المياه لاجيالها. المبحث الاول: الجدور التاريخيه للصراع على المياه بين العرب وإسرائيل: تعتبر المياه من المشكلات الدوليه المهوه، التي لها انعكاسات مباشرة على العلاهات بين الاول التي تثترك وي منابع ومصبات مياه الاتهار، خصوصا بعد دخول المياه إلى صلب النزاع في منطقه الثرق الاوسط والتي استعصت عن الحل لمدة عقود (1).

ان الجدور التاريخيه للصراع على المياه بين العرب وإسرائيل، ترجع في

الاساس إلى رغبه إسرائيل في السيطرة على مصادر المياه في المنطقه وهو حلهها القذيم الدي ربما ترجع بلايته إلى زمن ولادة المشروع الصهيوني نفسه، وقد وكر الصهاينه في بادئ الامر وي إحامه وطن وومي لليهود وي سيناء بعد تحويل نهر النيل

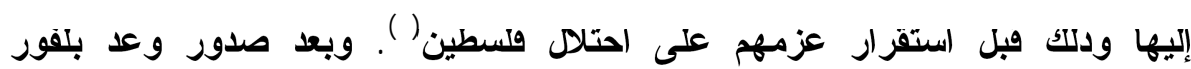
عام I9IV با الصهاينه يتحلتون بصوت عال عن حلمهم بإسرائيل الممتدة من النيل إلى القرات وهو اكبر مصدر للمياه والحياة في المنطقه. بل ان الزعيم الصهيوني حاييم وايزمان كتب في عام ·rوا إلى وزير الخارجيه البريطاني اللورد كيرزدت: ((ان الصهيونيه لا تريد ولسطين وحسب، بل تريد لحدودها ان تشمل ايضا جنوب لبنان ولا يكفي ان يكون الليطاتي واليرموك داخل الحدود بل من الضروري ضم الاراضي التي

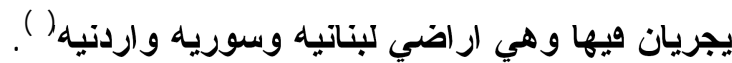

وبالنظر إلى ما توليه الصهيونيه من الهيه للمياه كعمود فقري للاستيطانه وقل اخدت الوكاله اليهوديه على عاتقها في البدء البلاد هلا الموضوع حقه من البحت و التتقيب وي سبيل الوصول إلى المبتغى، على هدا الاساس، انتابت هله الوكاله احد الخبراء الامريكيين في الجيولوجيات والمياه سنه 19 19، وهو " والتز كلاي لودر ميلك

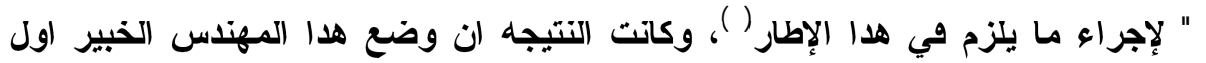
مخطط شامل لتنميه الموارد المائيه في ولسطين داخل الإطار السياسي والإتصادي 


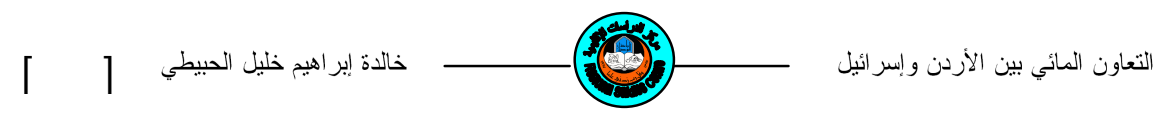

والامن العسكري للمطامع الصهيونيه في دوله يهوديها وكاتت التوصيات التي روقها لودرميلك و التي

الهيت حماس الاسرائليين القادة ويما بعد هو نشره للكتاب الدي يحمل عنوان

((ملسطين ارض الميعاد))فيسنة عالهاه ولا تزال حتى الان اساسا لجميع مشاريع إسرائيل المائيه(0). كما ان مشروع لودر ملك سيوفر في المستقبل المزارع والصناعه المانه والامن لما لا يقل عن اربعه ملايين لاجئ يهودي من اوربا بالإضاهه إلى مليون وتمانمئه الف نسمه من العرب واليهود موجودين وعلا في ولسطين وشرق الرردنه وتتمل احدى توصيات لودر ملك: ((ان لقلسطين حاجتين رئيستين: المياه والطاهه. والمياه متوفرة وي تدوق نهر الاردن، اما الطاهه الكامنه ومتوهرة في الاتحدار السريع

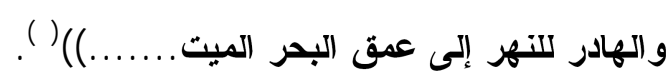
ولتوفر الاداة القانونيه الكلازمه بعد ويام الدوله الصهيونيه لتحقيق هده الاهداف، ونظر الإدراكها ان الموارد المائيه في ولسطين ستقل حتما عن سد حاجه كافها

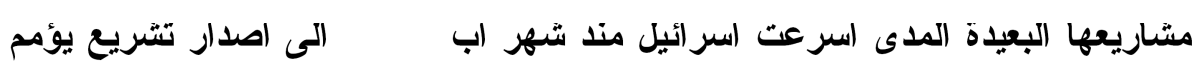
المياه وي البلاده ويقتن استتمارها، ويعتبرها كملك عام من حق الاوله وقط ان تتصرف بها لاغيا كل حق للاهر اد عليها، واناط هدا التشريع بوزير الزراعه مسؤوليه تنقيد احكامه ويساعده مفوض للمياه اوكلت إليه حصرا صلاحيه الترخيص لاي ورد او مجموعه من الاوراد بالحصول عن ايه كميه من المياه سواء من الاتهار او الينابيع او اوليه من حفر الابار (v). تانيا: النزاع بين إسرائيل والاردن حول مياه نهر الاردن:

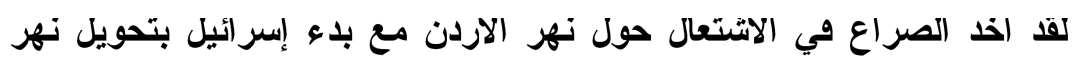

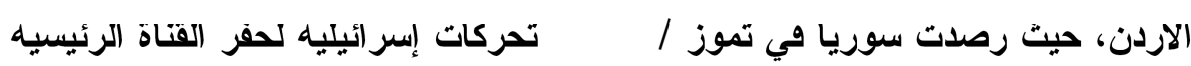
للمشروع بالقرب من حدودها في المنطقه المنزوعه السلاح شمال جربتات يعقوبه وسارعت سوريا بتقيم شكوى إلى مجلس الامن والبت بإصدار فرار للوفف القوري 
لهاه الاعمال، بعد رضض إسرائيل الاستجابه لاوامر كبير مراهبي الامم المتحدة

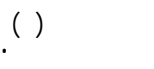

وازاء الموفف الجديد الدي هلد بعودة القتاله فام الرئيس الامريكي ايزنهاور

بتكليف المستشار جوتسون كمتل شخصي له بمهمه التقاوض مع دول المنطقه لمحاوله إفناعها بالمواققه على مشروع موحد لاستتمار الموارد المائيه في حوض نهر الاردن. ومع تقام مفاوضات جوتسون تضاءلت نقاط النزاع بين الاطراف العربيه و إسرائيل، اد وبل العرب باستخدام مياه النهر خارج الحوضه كما تنازلت إسرائيل عن مطالبتها بإدماج الليطاني في نظام نهر الاردن واقترحت الاطراف العربيه ان تكون بحيرة طبريا مركز تخزين لفائدة جميع الاطر اف وهو ما عارضته إسر ائيل، كدلك طالبت الاطراف الاردنيه بالإثراف الدولي على عليه توزيع المياها هان إسرائيل عارضت تلخل اي من اجهزة الامم المتحدة في موضوع استفلال المياه بين دول المنطقه. وبموجب خطه جوتسون حصلت إسرائيل على · . ع مليون متر مكعبه ونجا

ان مصر وإسرائيل ابديا القبول غير الرسمي بالتفاصيل الفتيه للخطه المقردة به(9)، وكان المشروعان الاساسيان هما: مشروع انبوب المياه الإسرائيلي، ومشروع هناة الخور الاردني.

ولكن عاد الصراع ليتفجر مرة اخرى فبيل انتهاء مشروع انبوب المياه الإسرائيلي وي عام 197ع، وقد تمت الدعوة لاول فمه عربيه لبحث مشكله نهر الاردن التي تقوم

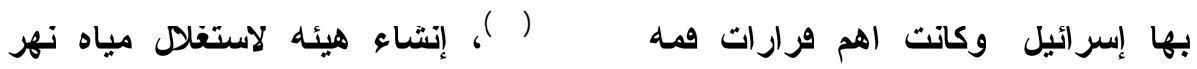
الاردنه وإقرار المشاريع اللازمه لاستتمار المياه العربيهه حيث اعتبر ها بديلا عن التذخل العسكري المباثر لإهشال خطط إسرائيله وكان امام الدول العربيه احد خيارين: ا - اما تحويل مياه الحصباني إلى الليطاني وتحويل مياه نهر بانياس الى لى اليرموك. م - او تحويل كل من الحاصباني وباتياس إلى اليرموك.

وتم الاستقرار على الخيار الاخير مع تخزين المياه خلف سد اهترح إقامته وهو سد

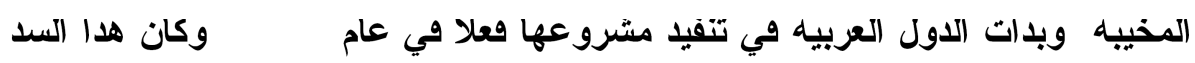




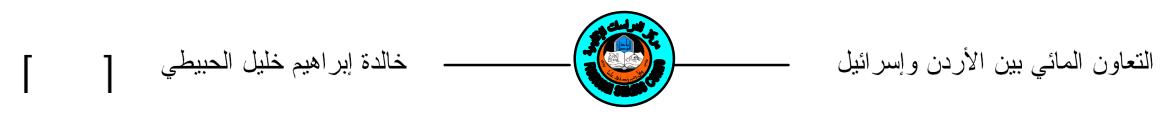

هو المشروع الاخطر على إسرائيل حيث يحجز رواد نهر الاردن عن التدهق إلى بحيرة طبريا هو (11). ولهلا اعلتت إسرائيل اتها لن تقف مكتوفه الايدي امام المشروعات العربيه، التي تلحق الضرر بها خاصه في ما يتعلق بسد المخيبه، الدي يعد وسيله تمد مصادر المياه التي تصل إلى إسرائيل، و إتها تعتبر هلا السد عملا من اعمال العدوانه وبالتالي هامت إسرائيل بشن هجمات استهروت مناطق العمل في المشروعه ووصلت الهجمات إلى اقصاها وي نيسان عام 197V، بتوجيه هجمه جويه في اعماق سوريا. وكاتت الصحف الإسر ائيليه ود دكرت ان النزاع حول مياه نهر الاردن يعتبر ورصه لإسر ائيل لثن الحرب في الوهت المناسب. وهناك من يرجح ان المياه كاتت ضمن الاسباب الرئيسيه لقيام

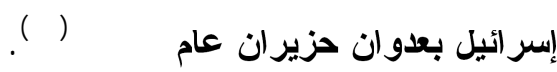
وكاتت النتائج المهمه لحرب $197 V$ عن جبهه المياه غايه وي الوضوح، وقا حسنت إسرائيل من موفعها المائي من خلال احتلال مرتقعات الجولان والضفه الغربيه. إد ان احتلال مرتفعات الجولان جعل من المستحيل على الدول العربيه تحويل مياه نهر الاردن مخطوط ووف إطلاق النار جعلت إسرائيل تتحكم في حوالي نصف طول نهر اليرموك مقارنه بحوالي · I كيلو مترات وقط فبل الحربه وهو ما جعل اي تنميه لنهر الاردن رهن وبضه إسر ائيل.

وبالتالي توفت اعمال سد المخيبه الدي كان مقررا ان يختزن . ..م مليون

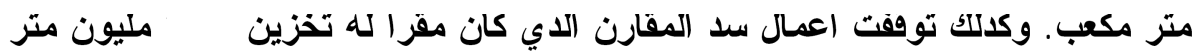
مكب. بينما توهقت المشروعات العربيهه هان إسرائيل ود اهيمت لها ورصه مناسبه

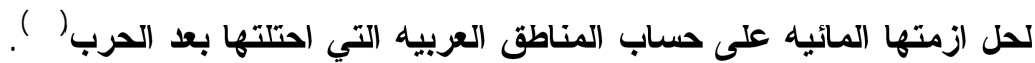


المبحث التاني: المفاوضات الاردنيه ـ الإسر ائيليه حول المياه:

بقيت المفاوضات الاردنيه ــ الإسر ائيليه حول مسالتي المياه والارضه تراوح

مكاتها خلال الاعوام الاخيرة إلى ان تسارعت وتيرتها وجاة واسقرت على توفيع معاهدة

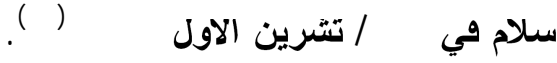

وكان المسؤولون الاردنيون في وهت وريب من المفاوضاته يعبرون عن خيبه

املهم من استمرار إسرائيل، خلال المفاوضات، في التعنت ازاء مساله الاراضي الاردنيه المحتله ومساكه تقاسم موارد المياه. وصد لاحظ المراقبون ان إسرائيل تتبنى مواقف متصلبه وتطالب بالحد الاوصى في المسائل المتعلقه بالمياه والحدود. وكانت تعد إلى المماطله في بحث هده المسائله في الوت الدي كان الاردن يصر على الإسراع في تحديد حصته المائيه وتعيين حدوده وهقا لمعاهدة الاتتاب الاردنيه البريطانيه في العام اعوا1ـ ومن الامور التي ادت إلى تعقيد المسالتين وجود اسرائلين على اراضي اردنبة هاموا بزراعتها، ووجود مياه جويه جارفه (اليرموك والاردن) على الحدود المشتركه (10).

وض تعترت المحادتات الاردنيه ـ الإسرائيليه، التي جرت وبل توفيع معاهدة

السلام بسبب الخلاف على نصيب الاردن من مياه نهري اليرموك والاردنه ومطالبته

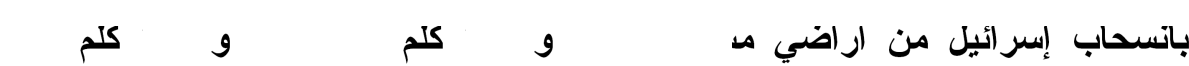
وادي عربه، جنوب البحر الميت و Ar و • كلم في الشمال ملتقي النهرين المدكورينه اي في المتلت الاردني ـ السوري ـ الإسرائيلي، و0 كلمّ بين بلاتي العقبه الاردنيه

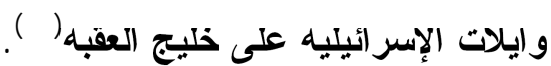
ان الجزء الاكبر من الاراضي الاردنيه المحتله يقع في منطقه تمتد من جنوب بحر الميت حتى خليج العقبه وتحتوي هده الاراضي على مياه جويه عميقه نسبيا تستفلها حاليا بعض المستعمرات الإسرائيليه وتضاف إليها فطعه ارض دات موهع

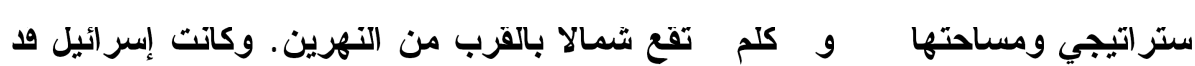
احتلت هده القطعه وي العام 19 19 بقصد السيطرة على ملتقى نهري اليرموك والاردن

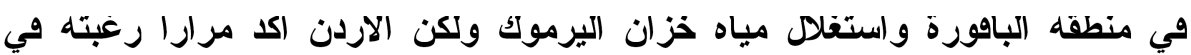




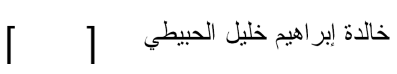

استعادة السيطرة على الممرات التي تفضي إلى النهرينه والاستفادة من حصته الضائعه في المياه، لان استرداد هله القطعه من الارض بسمح له باستفلال المياه العدبه من اليرموك وبل التقائه بنهر الاردنه جنوب بحيرة طبريا(IV). و إدا كان الاردن يطالب باستعادة الشريط الحدودي الممتد من البحر الميت الى الخليج العقبه ولان إسرائيل احتلت هده الاراضي الاردنيه بعد حرب $197 V$ وهي غير مشموله بقرار مجلس الامن روم كعr، الدي يقترض ان تجري على اساسه مفاوضات السلام العربيه ـ- الإسر ائيليه.

ويتراوح عرض الثريط المحتل بين . .م متر وش كلم وهو يتميز بوجود

مساحات زراعيه ويه وباتتثار مستعمرات إسرائيليه على مقربه منها والحقيقه ان إسرائيل اغتصبت هله الاراضي في العام • I9V بواسطه عمليات اشرف عليها ارييل شارون عندما كان فائدا للمنطقه الجنوبيه، وبدعوى حمايه الطريق الرئيسيه إلى ان تصل مرها ايلات بيقيه المناطق الإسرائيليه. واخيرا وفي مقابله مع صحيقه هارتس الإسرائيليه وي 199\%/1 1/1 1 اماط شارون التام عن الدواقع الكامنه وراء احتلال هله الاراضي وقال: اته لم يقم بلكك لمبررات امنيه وقط، بل لاته وضع في اعتباراته ايضا الاهيه الافتصاديه لتلك المنطقه بالنسبه إلى المستعمرات الإسرائيليه في وادي عربd (1) (11) والاهميه الاتصاديه لتلك المنطقه ليست وي الواقع سوى مائيها وقل فال المفوض الإسرائيلي لشؤون المياه، جدعون تسوده ان القرى الجماعيه والتعاونيه الواقعه في وادي عربه بين سدومه جنوب البحر الميث وايلات على الخليج العقبه، وعددها PO مستعرة، تستهلك نحو · ع مليون متر مكعب من المياه سنوياه منها 10 مليون تاتي من الاراضي الاردنيه. واكد المفوض ان هده الموارد ستزول "(د لبت

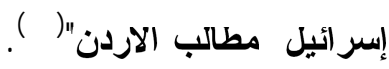


وبسبب الهيته العامل المائي اضطر رئيس الاستخبارات الإسرائيليه في الجيڤ، الجنرال اوري ساني، إلى روع تقرير الى لجنه الشؤون الخارجيه والدهاع وي الكنيست، وبل توفيع معاهدة السلام، جاء ويه: ((ان التوصل الى معاهاة سلام بين الاردن وإسرائيل لا يزال بعيدا كل البعد بسبب مشكله (المياه)). واعتبر المتحدث باسم الوض الإسرائيلي إلى محادتات السلامه يوسي غاله ان ضضيه المياه معقدة وتستلزم الكتير من العمل والجهد.

وشاطره الإثرار بها الواقع المرير المتحدث باسم الوود الاردني، مروان

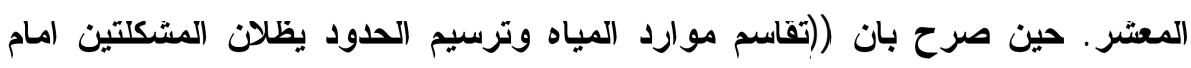
المفاوضين)). وكان رئيس الوض الاردني، وايز الطروانه يفكر في مشكله المياه عندما تحلث عند وجود وجوة في ما يتعلق بالمسائل الرئيسيه، في جذول اعمال المفاوضات(r). وعلى الرغم من هلا التصلب الظاهري والقلق المعلن، وقل دللت الصعاب وعقدت معاهدة السلام وتوصل الطرهان إلى حلول، في مسالتي الارض والمياه واستطاعت إسرائيل ان تلق مسمار وي الارض الاردنيه. وضد اكد العاهل الاردني الملك الحسين بن طلال، وي خطاب متلفزة القاه وي 1/10/199/1 ان معاهدة السلام مع إسرائيل اعادت إليه حقوفه المائيه في اليرموك والاردنه من احد الاطراف المعنيين بامر المياه [(إسرائيل)) والمح إلى ضرورة استرجاع الاردن حقوفه المائيه التي يستقلها

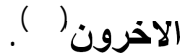

$$
\text { المبحث التالث: معاهدة السلام الاردني ـ الإسر ائيليه عام ع99 }
$$

ان الحرص الاردني على ترسيم الحدود ميع إسرائيل واهتسام مياه النهرين لم يلق تجاوبا في بنود المعاهدة. ودلك لان إسرائيل كانت ترغب في وضع اليا على المصادر العربيه وفي طليعتها نهر الاردن. وضد عبرت صحيفه جيروزليخ بوست

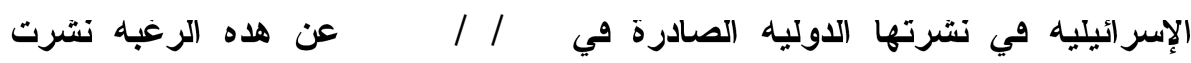

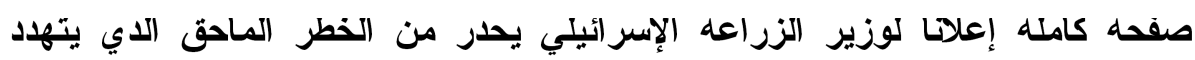




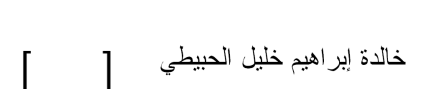

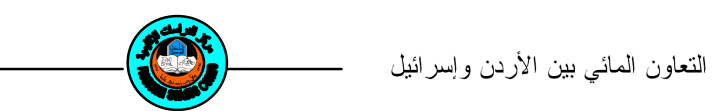

إسرائيل في حال انسحابها من الاراضي العربيه المحتله وتخليها عن مراقبه المياه وي جوارها.

وفد تضمن الملحق التاني من المعاهدة الاردنيه الإسرائيليه المتعلقه

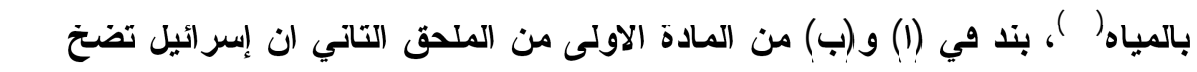
مليون متر مكب من مياه اليرموك في هترة الصيف الممتدة من 10 ايار حتى 10 Ir تشرين الاول من كل عامه ويحصل الاردن على بافي التدقى. كما تضخ إسرائيل مليون مّ"هي وترة الثتاء الممتدة من 17 تشرين الاول حتى عا ايار من كل عام. ويحصل الاردن على باهي التدوق.

ولكن الفقرة التانيه من البند (ب) تتضمن استتناء للالتزام الإسرائيلي الاي نص عليه مشروع حونستونه وقد ورد وي هاه الفقرة ان الاردن يواقق على ان تضخ

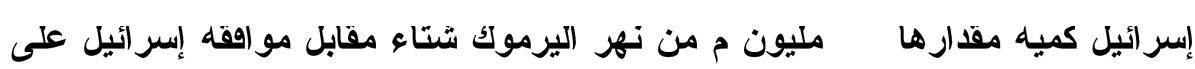
ان تقل كميه إضاهيه للاردن مقارها . م مليون م خلال هترة الصيف من نهر الاردن.

إضافه إلى دلك يحق لإسر ائيل المحافظه على استعمالاتها الحاليه لنهر الاردن، اما الاردن ويحق له الحصول على كميه مساويه لتلك التي تستعلها إسرائيل، على الا تضر استعمالاته بكميه المياه التي تستعملها إسر ائيل بنوعيتها (IT). وتحدد إسرائيل كميه إسيه المياه المحلاة التي يمكنه الحصول عليها من الينابيع المالحه المحوله إلى نهر الاردنه ويتعاون الطرهان على بناء سد تحويلي، وتخزيني على نهر اليرموكه وعلى إقامه نظام التخزين المياه على نهر الاردن (عان). وبالنسبه إلى الابار التي حفرتها إسرائيل واستعملتها والتي تقع على الجاتب الاردني من الحدود، هان إسرائيل تعترف بخضوعها للسيادة الاردنيهه ولكن المعاهدة تقر لإسرائيل بالاستمرار في استعماكها وتمنع الاردن من اتخاد اي إجراء من شانه 
التاتر، وي شكل ملحوظه في تقليل إنتاج هله الابار او في نوعيتها(ro). وحتى الابار القاشله فإنها تتعامل كما لو ان حقرها تم بموجب رخصه من الجهات الاردنيه المختصه وهت الحقر. وستقوم إسرائيل بتزويد الاردن بالبيانات الجيولوجيه والفتيه عن كل بئر ليصار إلى حفظها. وسيتم ربط البئر الجديد بانظمه (المياه والكهرباء الإسرائيليه)). (ما (تثغيل وصياته الابار وانظمتها الكواقعه وي الاراضي الاردنيه والتي تزود إسرائيل بالمياه، وكلتك انظمتها الكهربائيه فستكون من مسؤوليه الاردن)(T7). وهناك نص اخر في الملحق التاني يوحي بوجود حماسه إسرائيليه لحمايه مخصصات الاردن المائيه في مقابل تعهز اردني بحمايه حصه إسرائيل فالمادة التالته

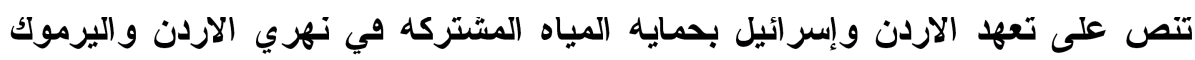
كل ضمن مناطق نفودها وكللك المياه الجوفيه في العربه إزاء اي تلوث وادى او

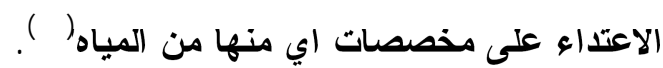

وعندما نطلع على تصريحات المسؤولين الاردنيين حول اهم ما ورد من مياه

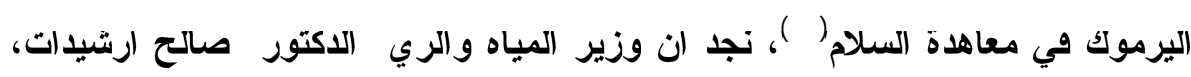

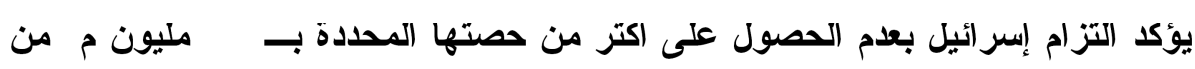

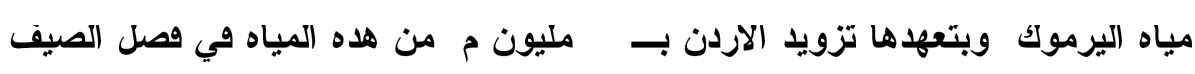
سنويا، وويامها بتخزين ·r مليون ممن مياه ويضان النهر سنويا لمصلحه الاردن

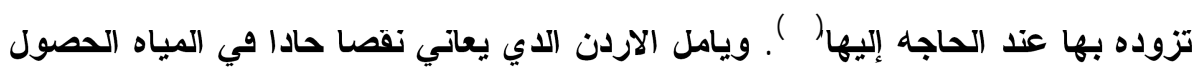

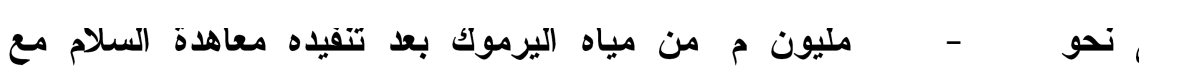
إسر ائيل(r)

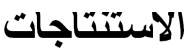

اولا: ان معاهدة السلام بين الاردن وإسرائيل لم تحقق كل تطلعات الاردن في الارض المياه. والملابسات التي اكتنقت توفيعها تتبا باستمرار إسرائيل في إتباع الاسلوب الاي يحقق اغراض استرتيجيتها العامها والتي تقوم على تحقيق مصاحها بشتى الوسائل و الطرق. 


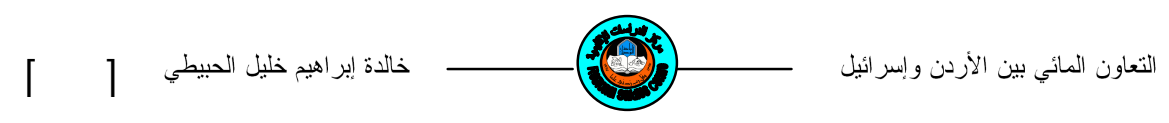

تانيا: نتيجه لاهيه المياه في الحاضر والمستقبل اصرت إسرائيل على إذخال بند المياه في اي مفاوضات تخوضها مع العربهوالغرض من دلك تامين المياه لنفسها بشكل كاف ومنتظم وإنـاع الانظمه العربيه بالتخلي عن الاعتبارات السياسيه لمصلحه الحسابات الافتصاديه والاخول ميع إسرائيل في مشاريع تتمويه مشتركه، كفيله بجعل المياه اساسا لارتباط إسرائيل عضويا بالشرق الاوسط واتدماجها في البيئه العربيه. تالتا: التاكيد على ان الصراع على المياه سيبقى في المنطقه مرتبطا بمدى صارة الدوله العربيه على حل المشكلات السياسيه وفي مقدمتها الصراع العربي - الإسرائليه قالصر اع المائي ود يتحوله في حاله استمرار الصراع السياسي إلى ازمه مزمنه تكون عو اوبها الاوتصاديه وخيمه جدا. رابعا: ان سعي إسرائيل إلى الاخول في معاهدات مع دول عربيه بما ان المياه يرجع إلى رغبتها في طرح مشاريع هي حلقه وي سلسله المشاريع المائيه التي اكل خبرائها الفتيون، مند عقود طويله، بهدف إقامه عوازل مائيه تحمي حدودها الحاليه من اي اجتياح عسكري مباغت وتحقيق ارتباط او تنابك بين مصاحها والمصالح العربيه يصعب التخلي عنه او التتصل منه في المستقبل. 


\title{
Water Cooperation Between Jordan And Israel And Peace Agreement of 1994
}

\author{
Khalida Ibrahim Khalil ${ }^{*}$
}

\begin{abstract}
Water Problem was and still one the main reasons of tension among states which have join water resources. Waters being the artery of life, then so many conflicts have risen among them to get the largest quantity of water.

Middle East region Arab Homeland in particular have witnessed a conflict between Arab countries and Israel about water. Israel has on putting the article of water in any negotiations made with the Arabs. The Purpose of this to ensure water for her and to convince Arab regimes to leave their Political considerations for their economic accounts. Thus, Water has become a basis for Israel to be a part of Middle East and to integrate with the Arab environment.
\end{abstract}

*Assisant Lecturer, Regional Studies Centre, Mosul University 
ا - زهر الاينه صالح، مشروع إسرائيل الكبرى بين الايموغرافيا والنفط والمياها.

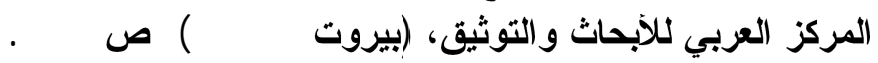

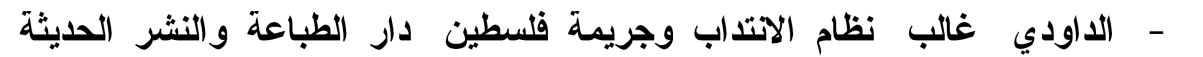

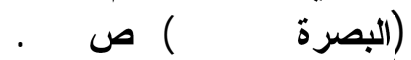

r - سليم احمد، دبلوماسية المياه أو الجديد في نهر الأردن، مجلة الهلال (القاهرة،

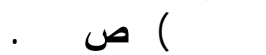
ع - عزيز، يحيى غالب، فلسطين ونهر الأردن،مطبعة (العاني،إبغداد، ع197)، ص . ع عزيز

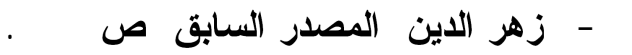

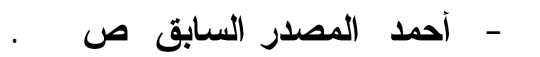

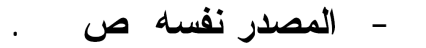

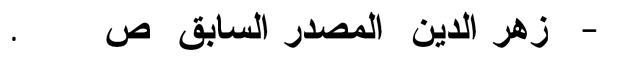

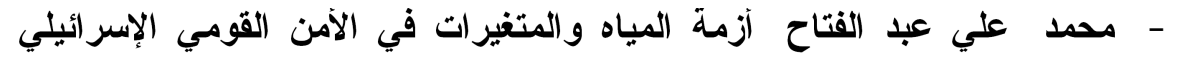

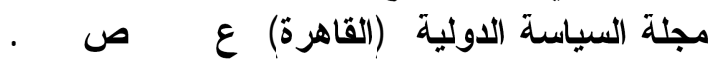

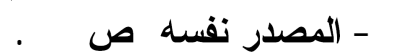

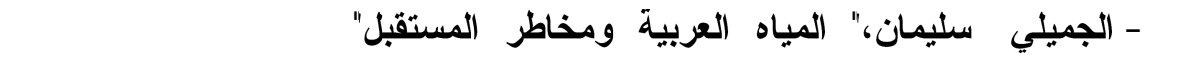

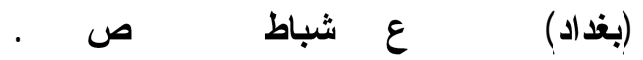

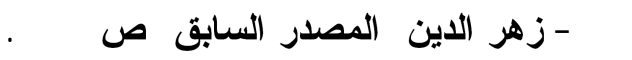

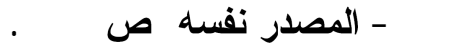

ع - المجذوب، طارق، لا احد يشرب، مشاريع المياه في ستر اتيجية إسر ائيل، (بيروت،

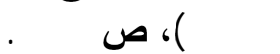

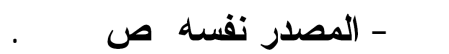

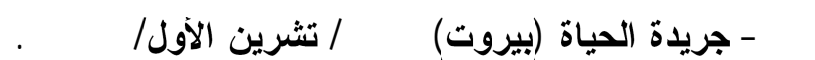
IV I 1 - المجذوب، المصدر نفية نفسهر ص - IV

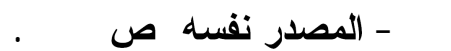

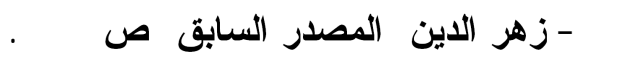


اب - المصدر نفسه/ ص MVO

مب - قاسم، عباس، الأطساع بالمياه العربية وأبعادها الجيولوجيتيكية، مجلة المستقبل

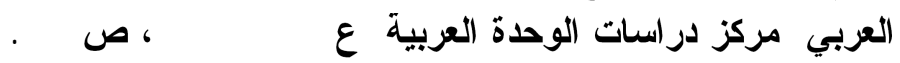

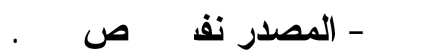

عr - عيس، نجيب مسالة المياه في الوطن العربي ومشروعات التكامل البديلة، مركز

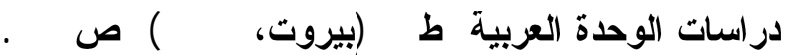

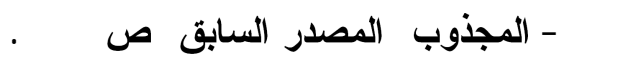

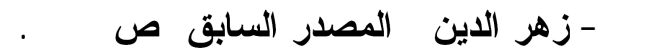

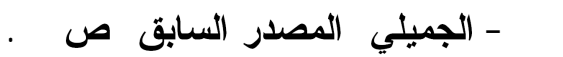

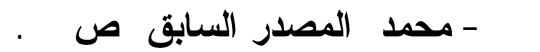

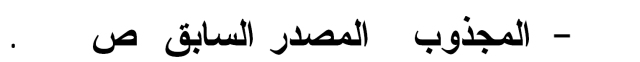

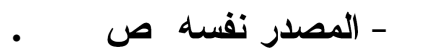

